\title{
Using mixed methods to better appreciate the life impact of upper limb reconstruction surgeries for tetraplegia in New Zealand - a study protocol
}

\author{
Kathryn Anne Sinnott Jerram ${ }^{1,2 *}$, Jennifer Ann Dunn ${ }^{3}$, Richard Peter Smaill ${ }^{2}$ and James Walter Middleton ${ }^{1,4}$ \\ ${ }^{1}$ John Walsh Centre for Rehabilitation Research, Sydney Medical School, Faculty of Medicine and Health, The University of Sydney, Australia \\ ${ }^{2}$ Burwood Academy of Independent Living, Christchurch, New Zealand \\ ${ }^{3}$ Department of Orthopaedic Surgery \& Musculoskeletal Medicine, University of Otago, Christchurch, New Zealand \\ ${ }^{4}$ Kolling Institute of Medical Research, Level 12, Royal North Shore Hospital, St Leonards, NSW 2065, Australia
}

\begin{abstract}
Background: Spinal cord injury (SCI) is a life altering event that causes sensorimotor and autonomic nervous system dysfunction, with wide ranging effects on health, functioning, participation and quality of life. Major damage to the cervical cord with complete tetraplegia produces the highest level and most severe impairment with the greatest disability. Restoration of arm/hand function in these circumstances involves multi-disciplinary rehabilitation to maximize ability as much as possible, in conjunction with use of adaptive technology and/or advanced reconstructive surgical interventions. Greater understanding of the process of decision-making at an early stage prior to full knowledge of life with tetraplegia, as well as the life impacts of surgical arm/hand reconstruction procedures is now more urgent given that the use of innovative nerve transfer surgeries is becoming more widespread, and by necessity are undertaken earlier (6-9 months post-injury) than conventional tendon transfers.

Methods: A multistage mixed methods convergent design is utilized to allow for concurrent analysis of the data for the three main patient reported outcome measures (PROMs) collected in New Zealand since 2010, comprising the qualitative content of the COPM and scores of the quantitative PWI and CUE-Qoutcome measures. Concurrently, a qualitative case series explores the lived experience of persons with tetraplegia at various time points between onset of SCI and either accepting or declining arm/hand reconstruction surgeries, including early NT procedures. The International Classification of Function Disability and Health (ICF) taxonomy is used as the analytical lens to guide data interpretation. Typology for the study is QUAL + QUAL $\rightarrow$ QUAN.

Discussion: From a health services perspective, this study series challenges the conduit role of research and lived experience collaborations embracing both rehabilitation and disability philosophies, to generate and translate knowledge in the field. First, there is a need for development of valid, reliable and sensitive PROMs that have patient-centered rather than clinician-directed origins. Second, there is a stronger demand for the involvement of consumers in SCI research to ensure that the voice of lived experience informs clinical practice, contributes to the research agenda that informs measurement development.
\end{abstract}

\begin{abstract}
Abbreviations: ICF: International Classification of Function Disability and Health; NT: Nerve Transfer; PROM: Patient reported outcome measures; SCI: Spinal Cord Injury.
\end{abstract}

\section{Introduction}

Spinal cord injury (SCI) involves damage to the central nervous system with severe bruising, laceration, or severing of the cord and/ or the associated effects of increased pressure, altered blood flow, hypoxia and cord perfusion following a sudden traumatic event or disease process, resulting in a variable loss of sensation, muscle paralysis and autonomic dysfunction below the level of injury [1] The level and extent (severity) of neurological impairment determines the residual functional capacity and muscles under voluntary control, with complete tetraplegia having the greatest degree of severity [2], and therefore the most disabling [3,4]. Yet there is little reference to 'disablement' in the field of arm/hand surgery research in the SCI [5,6]. Typically, comprehensive rehabilitation by an interdisciplinary specialist team focuses on physical retraining and exercise, developing new procedural skills along with compensatory strategies and use of adaptive equipment for independent living and self-management, as well as psychosocial support to assist adjustment and coping [7]. Research and innovation of new therapies, surgical approaches and advanced technologies have an important role to play in enablement, however, the various stakeholders involved in SCI research have valued functional outcomes differently with research often not being well aligned with the priorities of people with SCI [8]. For example, hope has been identified as a priority and an important facilitator of adjustment following SCI [9]. Recent findings suggest that contrary to the traditional perspective, high hope, even false hope, is associated with amongst other factors, greater acceptance of disability $[10,11]$.

With advances in research and technology over the latter part of the $20^{\text {th }}$ century more innovative interventions aimed at restoring upper extremity function for people with tetraplegia have evolved throughout

*Correspondence to: Kathryn Anne Sinnott Jerram, Burwood Academy of Independent Living, Private Bag 4708, Christchurch, New Zealand, Tel: +6421994878; E-mail: anne.sinnott@burwood.org.nz

Key words: mixed methods, tetraplegia, arm/hand surgery, research integrity

Received: March 18, 2019; Accepted: March 25, 2019; Published: April 04, 2019 
the world, with more options now being available for improving hand function and quality of life [12]. The hierarchy for surgical restoration of function begins with maximizing ability as much as possible based on the person's voluntary function, and then augmenting that function by implementing technology or advanced reconstructive surgical interventions [2,13-15]. Clinician-researchers in New Zealand have shown longstanding commitment in this field, contributing to advances in knowledge and practice. The contributions have included examining the decision-making processes faced by this small and vulnerable group of individuals with tetraplegia [16-21], use of the International Classification of Function, Disability and Health (ICF) [22] to interpret outcomes data [23] and the development of an international upper limb surgery registry for future data sharing given the world-wide challenges of small sample size for empirical research [24].

There is little published on the lived-experience of people with SCI following arm/hand surgery interventions that is considerate of the broader disability perspective. The justifications are wide-ranging, but in the first instance include the ethics of offering early elective surgery to people with tetraplegia before they can fully comprehend the life impacts of their disability, the validity of informed consent under such circumstances, the influence of clinician confidence on this process of decision-making and the longstanding voice of experience of life with tetraplegia. A traumatic cervical SCI results in varying degrees of loss of arm and hand function, depending on the level and extent of neurological damage, which is associated often with an inability to perform even basic activities of daily living, dependence on caregivers, differing levels of disability and reduced quality of life [12,25-28]. Baseline clinical assessments are routinely performed to classify the level and extent of neurological damage using two accepted international systems; the International Standards for Neurological Classification of SCI published by the American Spinal Injury Association (ASIA) as the ASIA Impairment Scale (AIS) [29] and the International Classification of Hand Surgery for Tetraplegia (ICSHT) [30]. These systems, and the complexities of rehabilitation to restore basic hand function following arm/hand surgical reconstructions have been well described and are essential to address for achieving best outcomes [25]. Furthermore, the limitations and difficulties in measuring and reporting outcomes following surgery have been acknowledged [25-27,31], providing an ongoing challenge. Despite efforts to classify level, extent and severity of injury a shared concern is the wide variety of measures that are used to determine outcomes. This problem is not restricted to this participant group or specific intervention alone, yet it is of fundamental importance when evidence suggests that improvements in function after surgical arm/hand reconstructions may only be relatively modest [21]. Until recently, surgical reconstructions were limited to the availability of innervated muscles for tendon transfer and/or functional electrical stimulation, but the use of innovative nerve transfer (NT) surgeries has become more widespread $[32,33]$. The time-limited nature of these new innovations, with need for early surgery ideally within the first 6-9 months post-injury, raises new challenges for clinicians in terms of accurate early prognostication, as well as for the potential surgery candidates, who may not yet have come to terms with the permanence of their disability [16-21]. In view of this requirement it is of interest that a recent review by Fox et al. [34] reports 'both nerve and tendon transfer surgeries produce gains in function and are valuable techniques that may be used alone or in combination. Augmenting the armamentarium of available treatment options provides individuals with choices that can be tailored to their goals and preferences'.

As innovations like elective early NT surgeries arise as treatment options for SCI there is a concomitant increase in the difficulties in developing and validating the most suitable outcome measures, with competing interests of different research groups favoring certain tools and a predominance of expert clinician-driven consensus in both design of measures [31] and the development of data sets [35,36]. While the various measures being used are considered to be the best available at the time, limitations may exist due to relatively little use of some in practice, and where data available for comparison is restricted to results from psychometric evaluation only [2,25-27,31]. The clinical utility, feasibility and sensitivity to detect functional changes must be reported in studies from independent sites to allow future improvement and/or development of new outcome measures to progress. Regardless of the type of innovation, and there will certainly be more coming in the future in SCI, patient-reported outcome measures (referred to as PROMs) are now being used more in research, clinical practice and management to assess how health services and interventions have impacted over time on attributes, which only patients can know, such as symptom severity, daily functioning, quality of life and other dimensions of health and wellbeing. Crucially, it is essential that the development phase for PROMs is well described and the fundamental differences between clinicianfocused and patient-centered origins are declared. This is important in the field of SCI rehabilitation, since the ultimate goal of treatment is not simply for individuals to survive and be able to function, but to thrive and enable full active participation, promoting high quality of life and adjustment to disability. Determining 'treatment benefit' is essential given the 'patient' is at the center of healthcare interventions. Pertinent to this question, PROMs have been described as unique indicators of the impact of disease on the patient, being helpful in the empowerment of the patients, necessary for determination of efficacy of the treatment, by communication helpful in creating a rapport between patient and healthcare providers, useful in the interpretation of clinical outcomes, treatment decision-making and establishing health care priorities for individuals living with SCI [8,37-39]. Yet the correlation between changes over time for PROMs and the lived-experience of arm/hand surgery for tetraplegia have not previously been reported. Qualitative data from interviews with individuals with tetraplegia tells us about their subjective experiences, interpretations, effects and life impacts of their SCI [4]. Thus, it can augment quantitative data in hypothesis generation, defining the actual interventions, functional questionnaire or instrument design by informing content, whereby fidelity of arm/ hand surgical intervention can be tested. This fidelity testing process has been described as "illuminating explanations (from qualitative data) for associations found within quantitative analysis" [40]. In addition to fidelity, feasibility is a consideration that requires a mixed method approach. It is not just about whether PROMs can be collected with missing data avoided, but how those measures are completed the validity of the data obtained. The qualitative data enables further refinement of the impact of the intervention, or not as the case may be, through feedback from mixed method findings. To this end, narrative data, identification of emerging themes and data fidelity will involve advice and input from the point of view of one of the current authors (RS), an academic with a health psychology background, who also has 44 years lived experience of tetraplegia, as well as being a recipient of hand surgery.

The overall objective of this multi-phased study is to determine convergence between clinician-directed PROMs and the voice from the lived-experience on the topic of complex elective arm/hand surgery interventions for individuals with tetraplegia. In particular, we emphasize the requirement for a pragmatic focus on design and data collection issues and data analysis. We would like to extend the literature through addressing these challenges as we attempt to 
explore the intersection between a specific intervention ( $\mathrm{arm} / \mathrm{hand}$ reconstructions), population (individuals with tetraplegia), and measurement integrity. The idea is for each study to complement and build upon one another to provide a deeper, enhanced appreciation of the life impacts of arm/hand reconstructions, more so than conducting a qualitative or quantitative investigation in isolation. It is believed that the flexibility provided by the mixed methods design allows us more scope to achieve these ends (Figure 1).

\section{Materials and methods}

\section{Overall design}

In order to answer the research questions above this project will implement a multistage mixed methods convergent design. Data stored in the New Zealand International Upper Limb Surgery Registry [24] since 2010, provides baseline data of SCI characteristics (including the ICSHT and AIS referred to above), as well as demographics and hand function using the gold standard grasp and release test (GRT) [41]. All participants are over the age of 16 years at time of clinical assessment and provide consent by an opt out option for data inclusion in the registry [24]. All data is collected routinely in the Burwood Spinal Unit Hand Clinic setting.

Objective 1: Identification of clinician-directed outcomes of interest: The focus of this phase is to ascertain the content and fidelity of the instruments used as per previously reported international consensus on measurement in this field [5]. Content analysis of the three arguably clinician-focused quantitative PROMs viewed through the lens of the ICF will be undertaken. The PROMs include the qualitative tasks/goals determined by participants using the Canadian Occupational Performance Measure [42], which provides access to the consumer voice and tasks/goals can be linked to the ICF taxonomy [43]. The remaining two PROMs are the Capabilities of Upper Extremity questionnaire [44] and the Personal Wellbeing Index [45]. The linking to the ICF taxonomy provides the analytical lens and underpins the coherence, enabling data from various sources to be merged. For example, linking the in-depth interview codes to ICF

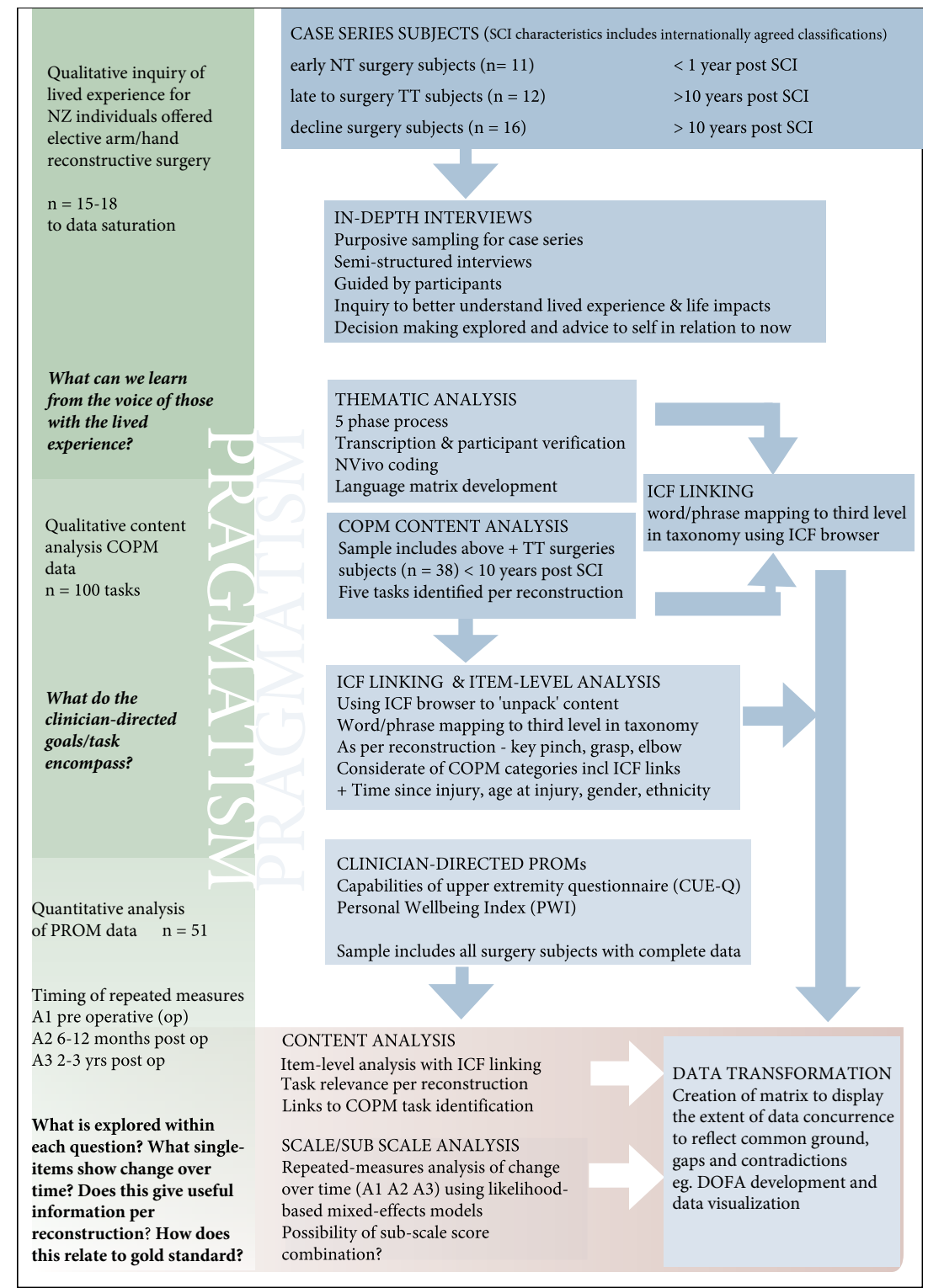

Figure 1. Overall study design 
categories, and linking the goal/task data to the ICF categories creates the possibility to look for conflicting data, with inconsistency or gaps. This process provides the opportunity for both content analysis and frequency of ICF linkages from both sets of data, while also comparing the ICF linkages to the content of the questions in terms of function and contextual factors. Concurrently, the quantitative scores can be analysed for strength of association with SCI characteristics, surgery details including bilateral/unilateral procedures, impairment scores, time since SCI and age.

Objective 2: Identification of life impacts of arm/hand surgery from the 'lived experience' perspective: The focus of this phase is to hear the voice of individuals living with tetraplegia who are clinically assessed as suitable for and subsequently offered elective arm/hand reconstructive surgery. To ensure representation purposive recruitment will create three participant groups: (i) individuals offered early NT procedures as part of the arm/hand reconstruction surgeries; (ii) late tendon transfer (TT) reconstruction recipients; and (iii) individuals who have declined surgery (DS). Semi-structured interviews using a guide with questions and prompts will be conducted face-to-face or via telephone, audio-recorded and transcribed verbatim, along with any field notes. Transcripts will be returned to participants for verification. Thematic analysis of data will be performed identifying codes and themes using NVivo [46], and matrix development based on a five-stage process. Sample size will be determined by reaching data saturation.

Objective 3: Translation of research knowledge into health services recommendations: The third objective of this study series is to translate knowledge gained from the previous qualitative and quantitative stages. This is essential as there is little point measuring clinician-directed outcomes if/when the content of the PROMs questions have not been scrutinized from a lived experience perspective. Theoretically, this integration process brings the QUANT numbers dataset and QUAL word datasets together for comparison in terms of content. This will be done by creating a data mapping system to ascertain the presence or absence of correlations between data sources. It is anticipated that a scoring system will be used to represent the greatest presence, absence or neutral agreement/mapping between data sources using a degree of freedom analysis (DoFA) [47]. Refer to (Figure 2) for the time frame overview, as per SPIRIT 2013 checklist item 13.

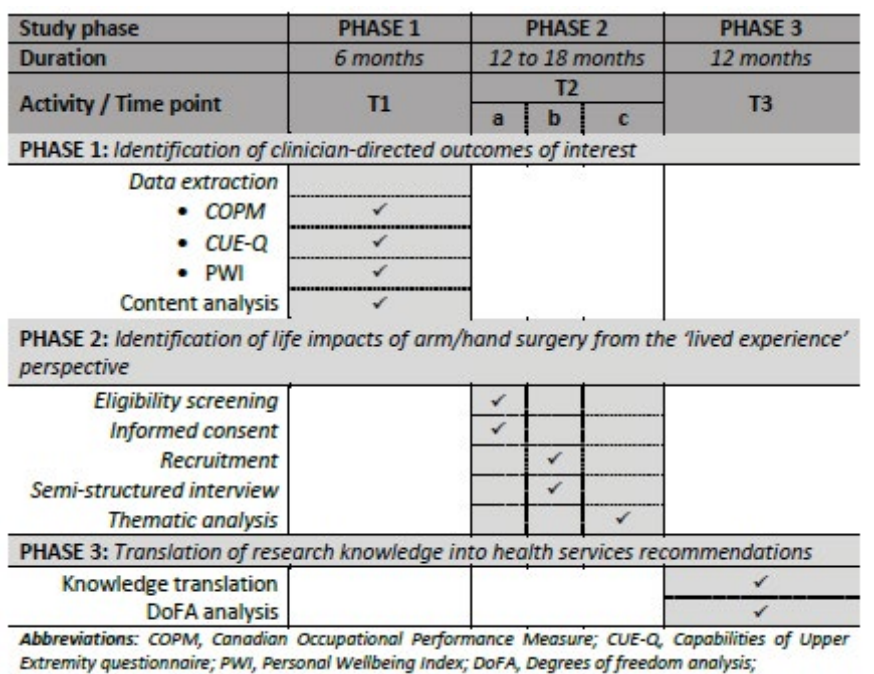

Figure 2. Spirit time schedule

\section{Discussion}

The findings of this study will contribute to a deeper appreciation of the life impacts of elective arm/hand surgery processes and procedures for individuals with tetraplegia. This is necessary to better inform the process of referral, assessment, evaluation, the potential range of measurement of outcomes and to facilitate international replication with more standardization, greater consistency and fidelity of the interventions through improved knowledge of the active component(s) and potential barriers to implementation. A mixed methods approach helps to answer whether an intervention works, why it works, and also its fidelity. Mixed methods have good potential in SCI research, since the majority of interventions are complex, whether surgical or non-surgical, and the process of evaluation and identification of suitable outcomes is particularly challenging $[4,26,48]$. We have adopted a pragmatic approach [49] with the design being driven by the nature and context of our research questions. For example, our sampling decisions relate to both the quantitative and qualitative components. For the quantitative component, we will be utilizing existing agreed PROMs data, which were recommended based on an expert therapist consensus group discussion. The data is stored in the New Zealand based International Upper Limb Surgery registry, which has previously been reported [24]. However, our choice of method has been driven not only by the research question/s, but also by the respondents targeted. For example, we were interested not only in the impact for newly injured individuals offered earlier NT surgeries within a time limited therapeutic window, but also the perspectives of those who have declined surgical reconstructions over time and individuals who made the decision to accept the offer of surgeries at a much later stage after onset of tetraplegia. Their livedexperience perspectives were also sought on the time limitations now imposed on decision-making to undergo NT procedures in newly injured individuals, given the obvious lack of experience of life with tetraplegia beyond the hospital doors. The challenge of data collection in this mixed methods design relates to the unique skills required for each method, and the need for flexibility within the mixed method framework. For example, there are certain sensitivities and empathy required by researchers working with vulnerable patients, often in their own homes. Yet at the same time, clinical challenges are apparent and resistance to the power of the lived-experience is evident in some clinical settings.

\section{Declarations}

\section{Ethical approval and consent to participat}

For the purpose of data collection an amendment to the existing 2014 New Zealand Health and Disability Ethics Committee (HDEC) data registry approval has been made to allow for the qualitative case series to be undertaken in New Zealand (14/NTB/46). This was approved in November 2016 for the purpose of this enquiry by Canterbury District Health Board Ethics Committee (RO 14063-A1). An extension to this time frame will be sought. Study information for participants has been provided to date, however, variations to the protocol are acceptable to the Canterbury District Health Board Ethics Committee based on peer review.

\section{Availability of data and material}

This is not a clinical trial. The New Zealand based International Upper Limb Surgery registry access was approved by the Canterbury District Health Board Ethics Committee. This registry is administered by the NZ Spinal Trust and is available for use with secure international 
web-based access. However, this is carefully controlled in New Zealand due to the small population and concerns regarding participant identification. De-identified data in SPSS format will be available from the corresponding author who has clinical-researcher access to all routinely collected measurement data entered as part of Hand Clinic assessments at Burwood Hospital, Christchurch, New Zealand.

\section{Competing interests}

There are no competing interests.

\section{Funding}

University of Sydney fees are covered by the Australian Clinical Training program. The data collection time-costs are not supported by salary, however travel costs are provided by the Upper Limb Surgery Endowment Fund administered by the NZ Spinal Trust (Charitable Foundation). The NZ Spinal Trust has no commercial interest in the data but do have an interest in the 'lived experience' contribution to SCI research. Their charitable trust status is external to the academic process of both the University of Sydney, Australia, and the Upper Limb Surgery research group, New Zealand.

\section{Author contributions}

The first author is undertaking this study series as her doctoral thesis project at the University of Sydney, Australia. The following three authors are academic supervisors. All authors contributed to the study design and development of this manuscript. JAD provided topic-field knowledge and clinical expertise in the tetraplegia and upper limb surgery field, outcome measurement expertise and gold standard measurement requirements. RPS provided the lived experience contribution to SCI research, mixed methods knowledge and recent methodological expertise. JWM provided support for overall study series structure development, meeting University of Sydney doctoral program requirements, peer review systems of study protocol review and scientific writing assistance. All co-authors contributed to the writing of this manuscript.

\section{Acknowledgments}

Our sincerest thanks to the study participants without whose candor this exploration would not be possible.

\section{Study status}

This study is ongoing. This protocol has been amended over two years and has been peer reviewed for the purpose of New Zealand Ethics Committee processes and University enrolment purposes. This paper is based on protocol version 3. From a mixed methods perspective the clinical PROMs data cut off point is yet to be reached. This is due to the low numbers and requirement for complete data sets across all time points. In addition, the first author has yet to complete the in-depth interviews, which for the early nerve transfer surgery participants recruitment is prospective. More significantly, the transformative phase of the analysis is under development and will be advanced in part as a result of the peer review process.

\section{References}

1. Bickenbach J, Biering-Sørensen F, Knott J, Shakespeare T, Stucki G, et al. (2013) Understanding spinal cord injury. International Perspectives on Spinal Cord Injury 1, Geneva: WHO. p. 1-9.

2. Fridén J, Gohritz A (2015) Tetraplegia Management Update. J Hand Surg Am 40: 24892500. [Crossref]
3. Bourke JA, Hay-Smith EJC, Snell DL, DeJong G (2015) Attending to biographical disruption: the experience of rehabilitation following tetraplegia due to spinal cord injury. Disabil Rehabil 37: 296-303. [Crossref]

4. Hammell KRW (2010) Spinal cord injury rehabilitation research: patient priorities, current deficiencies and potential directions. Disabil Rehabil 32: 1209-1218. [Crossref]

5. Sinnott KA, Dunn JA, Wangdell J, Johanson ME, Hall AS, et al. (2016) Measurement of Outcomes of Upper Limb Reconstructive Surgery for Tetraplegia. Arch Phys Med Rehabil 97: S169-181. [Crossref]

6. Sinnott KA, Brander P, Siegert RJ, Rothwell AG, De Jong G (2009) Life Impacts Following Reconstructive Hand Surgery for Tetraplegia. Topics in Spinal Cord Injury Rehabil 15: 90-97.

7. Kennedy P, Evans M, Sandhu N (2009) Psychological adjustment to spinal cord injury: the contribution of coping, hope and cognitive appraisals. Psychol Health Med 14: 1733. [Crossref]

8. Anderson KD (2004) Targeting recovery: priorities of the spinal cord-injured population. J Neurotrauma 21: 1371-1383. [Crossref]

9. Krause JS, Edles PA (2014) Injury perceptions, hope for recovery, and psychological status after spinal cord injury. Rehabil psychol 59: 176-182. [Crossref]

10. Dorsett P (2010) The Importance of Hope in Coping with Severe Acquired Disability Australian Social Work 63: 83-102.

11. Dorsett P, Geraghty T, Sinnott A, Acland R (2017) Hope, coping and psychosocial adjustment after spinal cord injury. Spinal Cord Ser Cases 3: 17046. [Crossref]

12. Bryden AM, Sinnott KA, Mulcahey MJ (2005) Innovative Strategies for Improving Upper Extremity Function in Person with Tetraplegia and Considerations in Measuring Functional Outcomes. Topics in SCI Rehabil 10: 75-93.

13. Freehafer AA (1991) Tendon transfers in patients with cervical spinal cord injury. $J$ Hand Surg Am 16: 804-809. [Crossref]

14. Moberg E (1975) Surgical treatment for absent single-hand grip and elbow extension in quadriplegia. Principles and preliminary experience. J Bone Joint Surg Am 57: 196206. [Crossref]

15. Zancolli EA (1975) Surgery for the quadriplegic hand with active, strong wrist extension preserved. Clin Ortho Rel Res 112: 101-113.

16. Mohammed KD, Rothwell AG, Sinclair SW, Willems SM, Bean AR (1992) Upper-limb surgery for tetraplegia. J Bone Joint Surg Br 74: 873-879. [Crossref]

17. Rothwell AG, Sinnott KA, Mohammed KD, Dunn JA, Sinclair SW (2003) Upper limb surgery for tetraplegia: a 10-year re-review of hand function. J Hand Surg Am 28: 489497. [Crossref]

18. Dunn JA, Hay-Smith EJ, Whitehead LC, Keeling S (2013) Liminality and decision making for upper limb surgery in tetraplegia: a grounded theory. Disab Rehabil 35: 1293-1301. [Crossref]

19. Dunn JA, Hay-Smith EJ, Keeling S, Sinnott KA (2016) Decision-making about upper limb tendon transfer surgery by people with tetraplegia for more than 10 years. Arch Phys Med Rehabil 97: 88-96. [Crossref]

20. Dunn JA, Rothwell AG, Mohammed KD, Sinnott KA (2014) The effects of aging on upper limb tendon transfers in patients with tetraplegia. J Hand Surg Am 39: 317-323. [Crossref]

21. Dunn JA, Sinnott KA, Rothwell AG, Mohammed KD, Simcock JW (2016) Tendon Transfer Surgery for People With Tetraplegia: An Overview. Arch Phys Med Rehabil 97: 75-80. [Crossref]

22. WHO (2001) International Classification of Functioning, Disability and Health: ICF, Geneva.

23. Sinnott KA, Dunn JA, Rothwell AG (2004) Use of the ICF conceptual framework to interpret hand function outcomes following tendon transfer surgery for tetraplegia. Spinal Cord 42: 396-400. [Crossref]

24. Sinnott KA, Dunn JA, Rothwell AG, Hall AS, Post MW (2014) The developmen of the NZ-based international upper limb surgery registry. Spinal Cord 52: 611-615. [Crossref]

25. Kalsi-Ryan S, Verrier M (2011) A synthesis of best evidence for the restoration of upperextremity function in people with tetraplegia. Physiother Can 63: 474-489. [Crossref]

26. Velstra I, Ballert C, Alarcos Cieza A (2011) A Systematic Literature Review of Outcome Measures for Upper Extremity Function Using the International Classification of Functioning, Disability, and Health as Reference. Phys Med Rehabil 3: 846-860. [Crossref] 
27. Connolly S, Aubut J, Teasell R, Jarus T, Team SR (2007) Enhancing upper extremity function with reconstructive surgery in persons with tetraplegia: a review of the literature. Topics in Spinal Cord Injury Rehabil 13: 58-80.

28. Charlifue S, Post MW, Biering-Sørensen F, Catz A, Dijkers M, et al. (2012) International Spinal Cord Injury Quality of Life Basic Data Set. Spinal Cord 50: 672675. [Crossref]

29. Kirshblum SC, Burns SP, Biering-Sorensen F, Donovan W, Graves DE, et al. (2011) International standards for neurological classification of spinal cord injury (Revised 2011). J Spinal Cord Med 34: 535-546. [Crossref]

30. McDowell CL, Moberg E, House JH (1986) The Second International Conference on Surgical Rehabilitation of the Upper Limb in Tetra-plegia (quadriplegia). J Hand Surg 11: 604-608.

31. Dunn JA, Sinnott KA, Bryden AM, Connolly SJ, Rothwell AG (2008) Measurement issues related to upper limb interventions in persons who have tetraplegia. Hand Clin 24: 161-168, v. [Crossref]

32. van Zyl N, Hahn JB, Cooper CA, Weymouth MD, Flood SJ, et al. (2014) Upper limb reinnervation in $\mathrm{C} 6$ tetraplegia using a triple nerve transfer: case report. $J$ Hand Surg Am 39: 1779-1783. [Crossref]

33. Cain SA, Gohritz A, Fridén J, Van Zyl N (2015) Review of upper extremity nerve transfer in cervical spinal cord injury. J Brachial Plex Peripher Nerve Inj 10: e34-e42. [Crossref]

34. Fox IK, Miller AK, Curtin CM (2018) Nerve and Tendon Transfer Surgery in Cervical Spinal Cord Injury: Individualized Choices to Optimize Function. Top Spinal Cord Inj Rehabil 24: 275-287. [Crossref]

35. Biering-Sorensen F, Bryden A, Curt A, Friden J, Harvey LA, et al. (2014) International Spinal Cord Injury Upper Extremity Basic Data Set. Spinal Cord 52: 652-657.

36. Biering-Sørensen F, Noonan VK (2016) Standardization of Data for Clinical Use and Research in Spinal Cord Injury. Brain Sci 6. [Crossref]

37. Organizations. IAoP (2007) What is Patient-centred Health Care? A Review of Definitions and Principles. London.
38. Simpson LA, Eng JJ, Hsieh JT, Wolfe D; Spinal Cord Injury Rehabilitation Evidence Scire Research Team (2012) The health and life priorities of individuals with spinal cord injury: a systematic review. J Neurotrauma 29: 1548-1555. [Crossref]

39. Bourke JA, Snell DL, Sinnott KA, Cassidy B (2012) A user-led consultation model making inclusion of service users in research a reality. Ethnicity and Inequalities in Health and Social Care 5: 61-69.

40. Farquhar MC, Ewing G, Booth S (2011) Using mixed methods to develop and evaluate complex interventions in palliative care research. Palliat Med 25: 748-757. [Crossref]

41. Wuolle KS, Van Doren CL, Thrope GB, Keith MW, Peckham PH (1994) Development of a quantitative hand grasp and release test for patients with tetraplegia using a hand neuroprosthesis. J Hand Surg Am 19: 209-218. [Crossref]

42. Law M, Baptiste S, McColl M, Opzoomer A, Polatajko H, et al. (1990) The Canadian Occupational Performance Measure: an outcome measure for occupational therapy. Can J Occup Ther 57: 82-87. [Crossref]

43. Cieza A, Geyh S, Chatterji S, Kostanjsek N, Ustün B, et al. (2005) ICF linking rules: an update based on lessons learned. J Rehabil Med 37: 212-218. [Crossref]

44. Marino RJ, Goin JE (1999) Development of a short-form Quadriplegia Index of Function scale. Spinal Cord 37: 289-296. [Crossref]

45. Cummins RA (2014) Personal Wellbeing Index. Deakin University.

46. Richards L (2005) Handling Qualitative Data: A Practical Guide. London: Sage Publications.

47. Tractenberg RE (2017) Degrees of Freedom Analysis in Educational Research and Decision-Making: Leveraging Qualitative Data to Promote Excellence in Bioinformatics Training and Education. Open Science Framework.

48. Elf M, Flink M, Nilsson M, Tistad M, von Koch L, et al. (2017) The case of value-based healthcare for people living with complex long-term conditions. BMC Health Serv Res 17: 24. [Crossref]

49. Greene J, Hall J (2010) Dialectics and pragmatism: being of consequence. In: Tashakkori A, Teddlie C, editors. Handbook of mixed methods in social and behavioral research. California, USA.

Copyright: (C2019 Jerram KAS. This is an open-access article distributed under the terms of the Creative Commons Attribution License, which permits unrestricted use, distribution, and reproduction in any medium, provided the original author and source are credited. 